\title{
The Survival of Patients With
} Alveolar Hemorrhage Secondary to
Antineutrophil Cytoplasmic
Antibody-associated Vasculitis
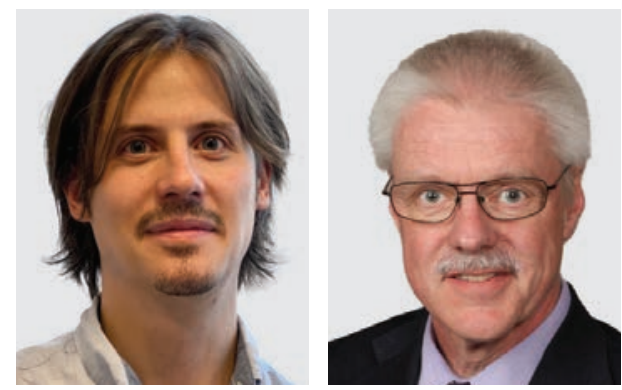

\author{
Alvise Berti ${ }^{1}$ (D) and Ulrich Specks ${ }^{2}$ (D)
}

Diffuse alveolar hemorrhage (DAH) is a clinical syndrome that can be caused by a variety of nonimmune-mediated and immune-mediated etiologies, including antineutrophil cytoplasmic antibody (ANCA)-associated vasculitis (AAV). DAH is one of the most severe and life-threatening manifestations of AAV, occurring in up to $25 \%$ of patients with $\mathrm{AAV}^{1}$. Some studies have suggested that DAH may be more common in microscopic polyangiitis (MPA) than in granulomatosis with polyangiitis (GPA), but reported frequencies may be affected by factors including variable epidemiology of MPA versus GPA in different regions of the world, applied disease definitions, and local referral patterns to tertiary care centers ${ }^{2,3,4,5}$. Preexisting airway disease has been reported as an independent risk factor for the development of $\mathrm{DAH}$ in $\mathrm{AAV}^{3}$. Irrespective of the frequency and severity of DAH in AAV, there is only a limited number of studies addressing the prognosis of DAH in patients with AAV (Table 1). The combination of glucocorticoids (GC) with cyclophosphamide (CYC) or rituximab (RTX), variably combined with plasma exchange (PLEX), is usually effective to induce remission of the disease in the majority of patients, and the main causes of death are infections or cardiovascular complications rather than vasculitis activity ${ }^{2,6,7,8,9,10}$.

${ }^{1}$ A. Berti, MD, Rheumatology Department, Santa Chiara Hospital and Department of Cellular, Computational and Integrative Biology (CIBIO), University of Trento, Trento, Italy, and Division of Pulmonary and Critical Care Medicine, Mayo Clinic College of Medicine and Science, Rochester, Minnesota, USA; ${ }^{2}$ U. Specks, MD, Division of Pulmonary and Critical Care Medicine, Mayo Clinic College of Medicine and Science, Rochester,

Minnesota, USA.

Dr. Specks received research grants from Roche/Genentech, ChemoCentryx, InflRx, GSK, and Astra Zeneca, and consulting fees from ChemoCentryx and Astra Zeneca.

Address correspondence to Dr. A. Berti, Rheumatology Department, Santa Chiara Hospital, Largo Medaglie D'Oro 9, 38121, Trento, Trentino, Italy. Email:alvise.berti@apss.tn.it.
In this issue of The Journal, Tang and colleagues ${ }^{11}$ report on their analysis of clinical features at presentation and the main prognostic factors of DAH in a single-center cohort of 92 patients with MPA. This is the largest cohort of DAH in patients with MPA published so far. Interestingly, in this cohort, the mortality was the highest reported for DAH in patients with AAV, with an overall survival of $63.7 \%$ and $47.3 \%$ at 1 year and 5 years, respectively. Multivariate analysis identified age $>65$ years, severe renal disease (serum creatinine $>500 \mathrm{umol} / \mathrm{L}$ ), ratio of partial pressure of arterial $\mathrm{O}_{2}$ to fraction of inspired $\mathrm{O}_{2}\left(\mathrm{PaO}_{2} /\right.$ $\left.\mathrm{FiO}_{2}\right)<300 \mathrm{mmHg}$, and involved lung area of $\geq 50 \%$ at presentation as independent risk factors for death in this cohort. The most frequent cause of death was infection, and $22 \%$ of patients died of active vasculitis. This report raises two questions: (1) Why was the mortality so much higher than that reported from other centers; and (2) was the mortality in these patients with MPA attributable only to DAH, or did other concomitant factors play a significant role?

Several factors should be considered as a possible explanation for the higher reported mortality in this cohort. In contrast to most previous reports from Western countries, the patients comprising this report are Chinese, and the different genetic background of these patients might have influenced the disease phenotype, the response to treatment, and ultimately the course of the disease ${ }^{12}$. All patients included in this study by Tang and colleagues ${ }^{11}$ had a diagnosis of MPA, whereas most other reported cohorts comprised a mix of MPA, GPA, and eosinophilic GPA (EGPA). Only one previous cohort included just patients with MPA, yet the 1-year and 5-year survival was $82 \%$ and $68 \%$, respectively ${ }^{13}$ (Table 1 ). In addition, being diagnosed with MPA, rather than GPA or EGPA, was not identified as a risk factor for death or for progression to respiratory failure in other cohorts of DAH in $\mathrm{AAV}^{2,8,14}$.

Similarly, $96 \%$ of the patients were positive for myeloper-

See MPA, DAH, and prognosis, page 410 
Table 1. The published cohorts of DAH occurring in patients with AAV (only studies with $\geq 20$ patients are reported).

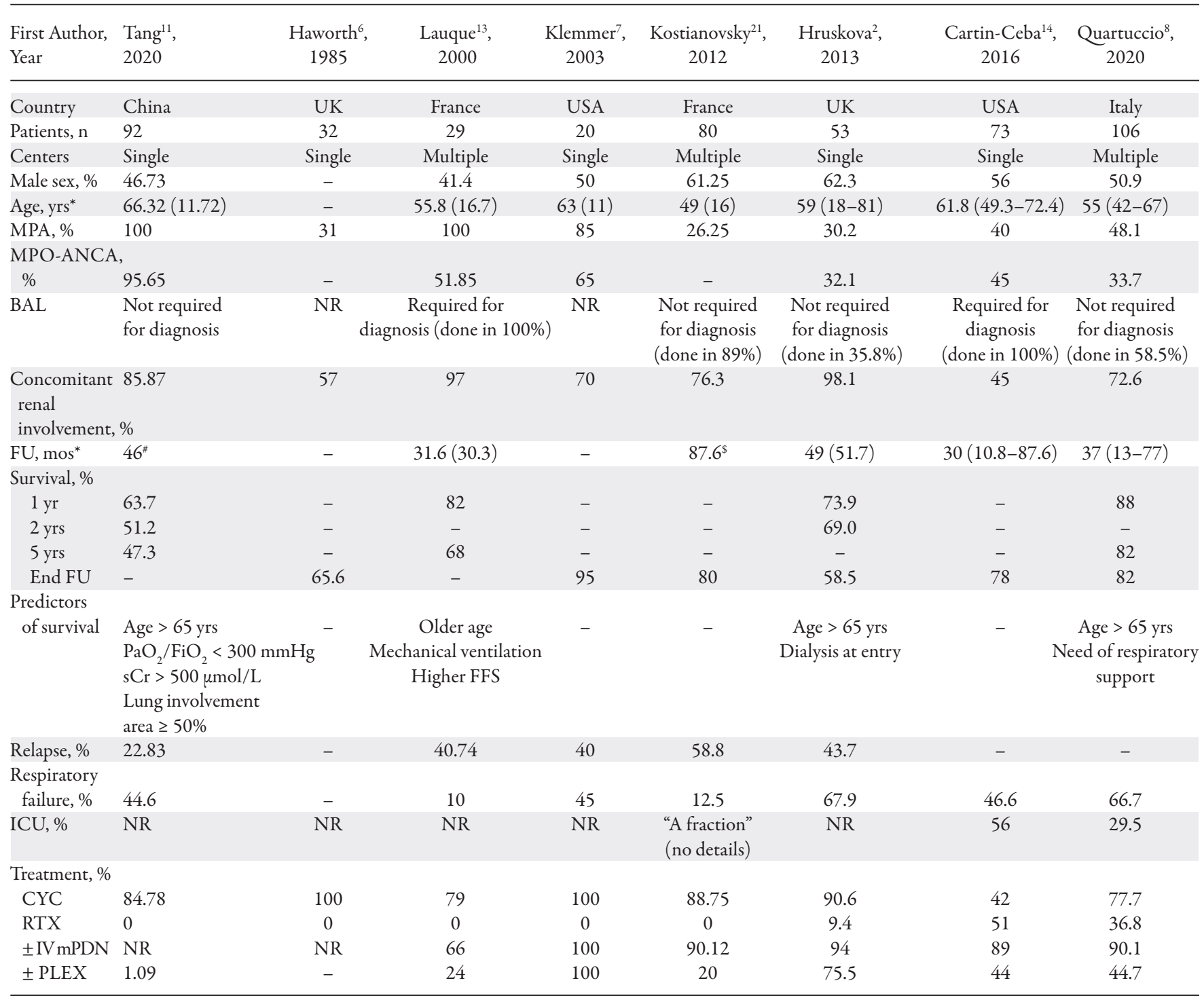

${ }^{*}$ Results are reported as median $[\mathrm{IQR}]$ or mean $(\mathrm{SD})$, according to the way in which they were reported in the paper. ${ }^{*}$ Median (no IQR was reported in the original article). ${ }^{\$}$ Mean (no SD was reported in the original article). AAV: antineutrophil cytoplasmic antibody-associated vasculitis; BAL: bronchoalveolar lavage; CYC: cyclophosphamide; DAH: diffuse alveolar hemorrhage; FFS: Five-Factor Score; FU: follow-up; ICU: intensive care unit; IV mPDN: intravenous methylprednisolone; MPA: microscopic polyangiitis; MPO-ANCA: myeloperoxidase antineutrophil cytoplasmic antibody; $\mathrm{NR}$ : not reported; $\mathrm{PaO} / 2 \mathrm{FiO}$ : ratio of partial pressure of arterial $\mathrm{O}_{2}$ to fraction of inspired $\mathrm{O}_{2}$; PLEX: plasma exchange; RTX: rituximab; sCr: serum creatinine.

oxidase (MPO)-ANCA. This is the highest seroprevalence of MPO-ANCA reported in any cohort of DAH. MPO-ANCA positivity is a known risk factor for death and endstage renal disease (ESRD) in $A A V^{15,16}$, and approximately $86 \%$ of patients in this cohort ${ }^{11}$ had concomitant renal involvement. This is probably explained by the close association between positive MPO-ANCA serology, glomerulonephritis, and clinical diagnosis of MPA. Together, these associations may have had a significant effect on the survival of the patients in this study, even though MPO-ANCA positivity [versus proteinase 3 (PR3)ANCA positivity] alone was not identified as a risk factor for progression to respiratory failure in $\mathrm{DAH}$ caused by $\mathrm{AAV}^{14}$. Of note, this cohort is also the one with the highest median age at diagnosis at 66.3 years $^{11}$ (Table 1). It is somewhat tautological that age is a risk factor for death, in both healthy individuals and patients with vasculitis ${ }^{17}$. Not surprisingly, older age is a risk factor for morbidity and mortality in patients with $A A V^{18}$, and it has been previously reported as a predictor of death in patients with $\mathrm{DAH}$ and $\mathrm{AAV}^{8,13}$. Considering that the age at diagnosis in patients with MPA is usually 10 years higher on average than patients with GPA (and similarly for patients with MPO-ANCA versus $P R 3-A N C A)^{19,20}$, the ultimate question is whether patients of this cohort had worse outcomes primarily because of the older age, or if other reasons should also be considered.

Other factors with a potential effect on reported outcomes of DAH in AAV are undoubtedly influenced by access to health- 
care, availability of intensive care, cultural attitudes toward intensive care in elderly patients with multiorgan failure, and local practice patterns. These factors lead to differences in time from disease onset to diagnosis (a median of 3.5 months in the Tang, et al study ${ }^{11}$ ) or initiation of definitive therapy, as well as differences in intensive care unit admission, which were not consistently reported in all the published cohorts.

The comparability of the different reports on DAH in $A A V$ is further complicated by different entry criteria applied across studies. For instance, the study by Tang, et al ${ }^{11}$ did not require the performance of bronchoscopy with bronchoalveolar lavage to confirm alveolar hemorrhage, as it was for a few of the previous studies on $\mathrm{DAH}^{13,14}$. Since $25-50 \%$ of patients with DAH do not have hemoptysis at the time of presentation ${ }^{11,14}$, it is conceivable that patients with different severity and acuity of DAH make up the various different cohorts.

Treatment is also heterogeneous across studies. In the Tang, et $a l^{11}$ study, the majority of patients were treated with GC and CYC; no patient received RTX, and approximately $18 \%$ of patients were treated with intravenous immunoglobulin (IVIG), which is not a generally accepted remission-induction treatment ${ }^{9,21}$. One could speculate that the use of IVIG to induce the remission of DAH in patients with AAV instead of CYC or RTX might be associated with an increased mortality. In contrast, the use of CYC instead of RTX cannot be blamed for the higher mortality of this cohort, as the overall prognosis reported for cohorts in which RTX was not used $\mathrm{d}^{6,7,13,21}$ was similar to those in which RTX was used ${ }^{2,8,14}$. Moreover, it was shown in the RAVE trial that patients with DAH at presentation $(\mathrm{n}=50)$ were as likely to respond to RTX as to $\mathrm{CYC}^{9}$. Notably, in the cohort reported by Tang and colleagues ${ }^{11}, 15 \%$ of patients did not receive intravenous methylpredinisolone (IV $\mathrm{mPDN}$ ) before starting oral GC because of side effects, concomitant infections, or $\mathrm{ESRD}^{8}$. Whether this affected mortality is unclear. In general, the great majority of patients with DAH receive IV mPDN before oral prednisone and immunosuppressive treatments for induction of remission ${ }^{2,7,8,13,14,21}$, and most guidelines advocate its use for patients with severe or life-threatening disease (i.e., DAH or severe renal disease) even though data on its benefit are acknowledged to be limited ${ }^{22}$. Unfortunately, the type of induction treatment was not included in the multivariate analysis of factors predicting survival in the study by Tang and colleagues ${ }^{11}$.

In all published cohorts except one ${ }^{6}$, PLEX was used in addition to standard immunosuppression for induction of remission in a variable fraction of patients, whereas only one patient was treated with PLEX in the cohort reported by Tang and colleagues $^{11}$. In 2016, Cartin-Ceba, et al reported no benefit of PLEX in patients with DAH due to AAV including those who required mechanical ventilation ${ }^{14}$. More recently, the role of PLEX has been investigated formally in a randomized controlled trial of 704 patients with severe AAV, of which 191 had DAH at presentation ${ }^{23}$. There was no observed benefit of PLEX on death and/or ESRD in this trial population or in the subset of patients with $\mathrm{DAH}^{23}$. It is therefore unlikely that the fact that PLEX was not used in the Tang, et al cohort affected the overall survival significantly ${ }^{11}$.
The study by Tang and colleagues in this issue of The Journal of Rheumatology provides a major, clinically relevant contribution because it analyzes risk factors for death in DAH caused by AAV in a careful multivariate analysis ${ }^{11}$. Previously identified risk factors including older age ( $>65$ years), respiratory insufficiency (assessed by the need for mechanical ventilation in most of the previous studies, or $\mathrm{PaO}_{2} / \mathrm{FiO}_{2}<300$ in the current one), and renal insufficiency (assessed as an increased serum creatinine or reduction of estimated glomerular filtration rate in most of the previous reports, or the need for hemodialysis at entry in the current study), were confirmed in this large cohort. In addition, a more extensive lung involvement by $\mathrm{DAH}$, estimated by radiographic imaging ( $50 \%$ or more) is reported here for the first time as an independent predictor of survival in AAV (Table 1). Even if most of the risk factors for death identified by this study confirm the results of previously published reports ${ }^{2,6,7,8,13,14,21}$, it is worth noting that there is significant heterogeneity in the way risk factors are assessed across different studies. Thus, confirmatory results obtained with complementary methods of analysis provide additional confidence in the findings for clinicians. Finally, other outcomes generally reported in studies focusing on DAH in patients with AAV include respiratory failure and relapse rate. In the Tang, et al study ${ }^{11}$, respiratory failure occurred in $44.6 \%$ and relapse in $22.83 \%$ of patients. Whereas respiratory failure varies among different studies, ranging from $10 \%$ to $67.9 \%$, reported relapse rates seem to correlate inversely with the percentage of patients with a diagnosis of MPA (versus GPA) or MPO-ANCA positivity (versus PR3-ANCA positivity). Ultimately, it is difficult to identify and compare the contributors to mortality across studies because of the substantial variability in diagnosis and management of $\mathrm{DAH}$ in $\mathrm{AAV}$, as well as variability of study inclusion criteria and composition of patient cohorts across the globe.

In conclusion, the overall mortality of $\mathrm{AAV}$ patients presenting with DAH remains high, but active vasculitis is the cause of death in only a minority of cases, whereas the majority of patients die from severe infections and cardiovascular complications. One can only speculate which factors might have contributed to the mortality being higher compared to previous studies, as reported in this study in The Journal ${ }^{11}$. The most plausible explanation is the older age of the patients at diagnosis, reflecting the median age at diagnosis of patients with MPA. Delays in diagnosis and initiation of treatment as well as the treatment choice, particularly the use of IVIG instead of standard immunosuppression in a sizable fraction of patients, may also have been factors. However, this is contrasted by the fact that the rate of respiratory failures as well as the major identified risk factors for death are in line with previous publications. This study provides precious insights into a rare medical condition. At the same time, it illustrates the difficulties arising from the retrospective nature of cohort studies, which make their data so difficult to compare. This calls for harmonization of the way in which studies evaluating DAH in patients with AAV are conducted, and for a shared strategy for the management of $\mathrm{DAH}$ in the context of AAV, with the ultimate goal to reduce

Personal non-commercial use only. The Journal of Rheumatology Copyright (c) 2021. All rights reserved. 
the early mortality of this syndrome. Early diagnosis and implementation of effective therapy for DAH remains the most significant challenge.

\section{REFERENCES}

1. Thompson GE, Specks U. Update on the management of respiratory manifestations of the antineutrophil cytoplasmic antibodies-associated vasculitides. Clin Chest Medicine 2019; 40:573-82.

2. Hruskova Z, Casian AL, Konopasek P, Svobodova B, Frausova D, Lanska V, et al. Long-term outcome of severe alveolar haemorrhage in ANCA-associated vasculitis: a retrospective cohort study. Scand J Rheumatol 2013;42:211-4.

3. Kida T, Tanaka T, Yokota I, Tamagaki K, Sagawa T, Kadoya M, et al. Association between preexisting lung involvements and the risk of diffuse alveolar hemorrhage in patients with microscopic polyangiitis: a multi-center retrospective cohort study. Mod Rheumatol 2020;30:338-44.

4. Mohammad AJ, Mortensen KH, Babar J, Smith R, Jones RB, Nakagomi D, et al. Pulmonary involvement in antineutrophil cytoplasmic antibodies (ANCA)-associated vasculitis: the influence of ANCA subtype. J Rheumatol 2017;44:1458-67.

5. Solans-Laque R, Fraile G, Rodriguez-Carballeira M, Caminal L, Castillo MJ, Martinez-Valle F, et al; Spanish Registry of systemic vasculitis (REVAS) from the Autoimmune Diseases Study Group (GEAS) of the Spanish Society of Internal Medicine (SEMI). Clinical characteristics and outcome of Spanish patients with ANCA-associated vasculitides: impact of the vasculitis type, ANCA specificity, and treatment on mortality and morbidity. Medicine 2017;96:e6083.

6. Haworth SJ, Savage C, Carr D, Hughes JM, Rees AJ. Pulmonary haemorrhage complicating Wegener's granulomatosis and microscopic polyarteritis. Br Med J 1985;290:1775-8.

7. Klemmer PJ, Chalermskulrat W, Reif MS, Hogan SL, Henke DC, Falk RJ. Plasmapheresis therapy for diffuse alveolar hemorrhage in patients with small-vessel vasculitis. Am J Kidney Dis 2003; 42:1149-53.

8. Quartuccio L, Bond M, Isola M, Monti S, Felicetti M, Furini F, et al; Italian Study Group on Lung Involvement in Rheumatic Diseases and the Italian Vasculitis Study Group. Alveolar haemorrhage in ANCA-associated vasculitis: long-term outcome and mortality predictors. J Autoimmun 2020;108:102397.

9. Stone JH, Merkel P a, Spiera R, Seo P, Langford C a, Hoffman GS, et al; RAVE-ITN Research Group. Rituximab versus cyclophosphamide for ANCA-associated vasculitis. N Engl J Med 2010;363:221-32.

10. Specks U, Merkel PA, Seo P, Spiera R, Langford CA, Hoffman GS, et al; RAVE-ITN Research Group. Efficacy of remission-induction regimens for ANCA-associated vasculitis. N Engl J Med 2013;369:417-27.
11. Tang S, Li X, Zhao K, Zhou Q, Tang X. Clinical characteristics and prognostic analysis of microscopic polyangiitis with diffuse alveolar hemorrhage. J Rheumatol 2021;48:410-16.

12. Chang DY, Luo H, Zhou XJ, Chen M, Zhao MH. Association of HLA genes with clinical outcomes of ANCA-associated vasculitis. Clin J Am Soc Nephrol 2012;7:1293-9.

13. Lauque D, Cadranel J, Lazor R, Pourrat J, Ronco P, Guillevin L, et al. Microscopic polyangiitis with alveolar hemorrhage. A study of 29 cases and review of the literature. Groupe d'Etudes et de Recherche sur les Maladies “Orphelines" Pulmonaires (GERM”O”P). Medicine 2000;79:222-33.

14. Cartin-Ceba R, Diaz-Caballero L, Al-Qadi MO, Tryfon S, Fervenza FC, Ytterberg SR, et al. Diffuse alveolar hemorrhage secondary to antineutrophil cytoplasmic antibody-associated vasculitis: predictors of respiratory failure and clinical outcomes. Arthritis Rheumatol 2016;68:1467-76.

15. Mohammad AJ, Segelmark M. A population-based study showing better renal prognosis for proteinase 3 antineutrophil cytoplasmic antibody (ANCA)-associated nephritis versus myeloperoxidase ANCA-associated nephritis. J Rheumatol 2014;41:1366-73.

16. Berti A, Cornec D, Crowson CS, Specks U, Matteson EL. The epidemiology of ANCA associated vasculitis in Olmsted County, Minnesota: a 20 year population-based study. Arthritis Rheumatol 2017;69:2338-50.

17. Guillevin L, Pagnoux C, Seror R, Mahr A, Mouthon L, Le Toumelin P; French Vasculitis Study Group (FVSG). The Five-Factor Score revisited: assessment of prognoses of systemic necrotizing vasculitides based on the French Vasculitis Study Group (FVSG) cohort. Med 2011;90:19-27.

18. Berti A, Felicetti M, Monti S, Ortolan A, Padoan R, Brunori G, et al. Disease and treatment-related morbidity in young and elderly patients with granulomatosis with polyangiitis and microscopic polyangiitis. Semin Arthritis Rheum 2020 Feb 24 (E-pub ahead of print).

19. Cornec D, Cornec-Le Gall E, Fervenza FC, Specks U. ANCA-associated vasculitis - clinical utility of using ANCA specificity to classify patients. Nat Rev Rheumatol 2016;12:570-9.

20. Berti A, Caporali R, Montecucco C, Paolazzi G, Monti S. Aging in primary systemic vasculitis: implications for diagnosis, clinical manifestations, and management. Drugs Aging 2019;36:53-63.

21. Kostianovsky A, Hauser T, Pagnoux T, Cohen P, Daugas E, Mouthon L, et al. Alveolar haemorrhage in ANCA-associated vasculitides: 80 patients' features and prognostic factors. Clin Exp Rheumatol 2012;1 Suppl 70:S77-82.

22. Geetha D, Jin Q, Scott J, Hruskova Z, Hanouneh M, Little MA, et al. Comparisons of guidelines and recommendations on managing antineutrophil cytoplasmic antibody-associated vasculitis. Kidney Int Rep 2018;3:1039-49.

23. Walsh M, Merkel PA, Peh CA, Szpirt WM, Puechal X, Fujimoto S, et al; PEXIVAS Investigators. Plasma exchange and glucocorticoids in severe ANCA-associated vasculitis. N Engl J Med 2020; 382:622-31. 